\title{
Limit Cycle and Conserved Dynamics
}

\author{
X.-M. Zhu' ${ }^{1)}$, L. Yin ${ }^{2)}$, and P. Ao ${ }^{3)}$ \\ 1) GenMath, 5525 27th Ave. N.E., Seattle, WA 98105, USA \\ ${ }^{2)}$ School of Physics, Peking University, 100871 Beijing, PR China \\ ${ }^{3)}$ Department of Mechanical Engineering, University of Washington, Seattle, WA 98195, USA
}

(Dated: Dec. 7, 2004)

\begin{abstract}
We demonstrate that a potential coexists with limit cycle. Here the potential determines the final distribution of population. Our demonstration consists of three steps: We first show the existence of limit from a typical physical sciences setting: the potential is a type of Mexican hat type, with the strength of a magnetic field scale with the strength the potential gradient near the limit cycle, and the friction goes to zero faster than the potential near the limit cycle. Hence the dynamics at the limit cycle is conserved. The diffusion matrix is nevertheless finite at the limit cycle. Secondly, we construct the potential in the dynamics with limit cycle in a typical dynamical systems setting. Thirdly, we argue that such a construction can be carried out in a more general situation based on a method discovered by one of us. This method of dealing with stochastic differential equation is in general different from both Ito and Stratonovich calculus. Our result may be useful in many related applications, such as in the discussion of metastability of limit cycle and in the construction of Hopfield potential in the neural network computation.
\end{abstract}

\section{INTRODUCTION}

Noise perturbed dynamics systems with limit cycles are abounded in physical sciences. It is one of the driving forces in the study of nonlinear systems ${ }^{1.2}$. Until recently, one of the most useful concepts in physical sciences, the potential energy function, had been found to be not applicable in general ${ }^{2.3}$. Hence, it has been concluded that the no Lyapunov function or potential function with periodic attractors, in the neural network computing ${ }^{4}$. Similar statements can also be found in many other fields. When the matastability and the decay of metability state are discussed, efforts have been made to avoid the use of potential energy function in the form of Boltzmann-Gibbs distribution. Various methods which can go around this issue, such as the MachlupOnsager functional method, quasipotentials, etc, have been developed ${ }^{5}$. They have been successfully applied to situations with limit cycle dynamics, such as in the discussion of topological structure of the decay of metastable limit $\operatorname{cycle}^{6}$, in the numerical study of trajectory of the escaping path ${ }^{7}$. However, even in such settings, there is a need to consider potential energy function, such as indicated in the escape of asteroids in the solar system ${ }^{8}$. Hence, it is a natural question to ask what would be the connection between such an approach and the classical approach based on potential energy function ${ }^{9.10}$, and whether or not the potential function exists in dynamics with limit cycle. The purpose of the present article is to give an explicit demonstration that at least for the case of limit cycle dynamics, potential function can be explicitly constructed, which provides a critical link between those two ways of dealing with metastability.

Our construction is based on a novel way of handling the stochastic differential equation. It can be understood as the zero mass limit of a generalized Klein-Kramers equation ${ }^{2}$. In this limit the steady state distribution can be established from the Klein-Kramers equation with- out the concerns of the Ito or Stratnovich calculus. In this sense it would not be surprising that a different perspective can be obtained other than those in Ref.[6.7.8]. We believe that our following demonstration provides an important step for an alternative understanding of the nonequilibrium processes such as limit cycles.

We will demonstrate the co-existence of a potential with limit cycle in three steps in three sections, respectively. In section II we explicitly demonstrate how to construct a limit cycle based on our knowledge in physical sciences, with a potential. This is different from the usual approach in dynamical systems. In section III we demonstrate how to construct a potential in the presence of a limit cycle in the dynamical systems setting. In section IV we outline a general construction of potential in a broad class of dynamical systems, including limit cycles. In section $\mathrm{V}$ we discuss two mathematical subtleties. In section VI we conclude that the potential can co-exist with limit cycle and with possibly more complicated dynamics.

\section{LIMIT CYCLE: PHYSICAL SCIENCES POINT OF VIEW}

In physical sciences, the general dynamical equation for a massless particle in two dimensional state space may be expressed as, with both deterministic and stochastic forces 11 :

$$
[S(\mathbf{q}, t)+T(\mathbf{q}, t)] \dot{\mathbf{q}}=-\nabla \psi(\mathbf{q}, t)+\xi(\mathbf{q}, t),
$$

and supplemented by the relationship on the stochastic force:

$$
\left\langle\xi(\mathbf{q}, t) \xi^{\tau}\left(\mathbf{q}, t^{\prime}\right)\right\rangle=2 S(\mathbf{q}, t) \epsilon \delta\left(t-t^{\prime}\right),
$$

and $\langle\xi(\mathbf{q}, t)\rangle=0$. Here $\mathbf{q}^{\tau}=\left(q_{1}, q_{2}\right)$ with $q_{1}, q_{2}$ the two Cartesian coordinates of the state space, which may be 
perceived as the position space of the massless particle. The transpose is denoted by the superscript $\tau$, and $\dot{\mathbf{q}}=$ $d \mathbf{q} / d t$.

The scalar function $\psi$ will is the usual potential energy function. Its graphical representation in the state space is a landscape. The antisymmetric matrix $T$ represents the dynamics which conserves the potential, corresponding to the Lorentz force in physical sciences, determined by the magnetic field. The matrix $S$ represents the dynamics which decreases the potential, the dissipation. This matrix will be called friction matrix. The friction matrix is connected to the stochastic force $\xi$ by Eq.(2), which guarantees that it is nonnegative and symmetric. All $T, S, \psi$ can be nonlinear functions of the state variable $\mathbf{q}$ as well as the time $t$. The numerical parameter $\epsilon$ corresponds to an effective temperature, which will be taken to be zero to recover the deterministic dynamics. It has been shown that if a steady state distribution $\rho(\mathbf{q})$ in state space exists,

$$
\rho(\mathbf{q}) \propto \exp \left(-\frac{\psi(\mathbf{q})}{\epsilon}\right)
$$

a Boltzmann-Gibbs type distribution function ${ }^{2}$. Eq.(3) implies that for dynamics which repeats itself indefinitely, such as limit cycle, the potential may be the same along such trajectory.

It should be pointed that the potential function $\psi(\mathbf{q}, t)$ exists from the beginning by construction. This is one of most useful quantitative concepts in physical sciences. If the stochastic force could be set to be zero, $\xi(\mathbf{q}, t)=0$, that is, the deterministic dynamics, the dynamics of this massless particle always decreases its potential energy:

$$
\begin{aligned}
\dot{\mathbf{q}}^{\tau} \nabla \psi(\mathbf{q}, t) & =-\dot{\mathbf{q}}^{\tau}[S(\mathbf{q}, t)+T(\mathbf{q}, t)] \dot{\mathbf{q}} \\
& =-\dot{\mathbf{q}}^{\tau} S(\mathbf{q}, t) \dot{\mathbf{q}} \\
& \leq 0 .
\end{aligned}
$$

The zero occurs only at the limiting set. Hence the potential function has the usual meaning of Lyapunov function.

To model a limit cycle, we choose following forms for the friction matrix $S$, the anti-symmetric matrix $T$, and the potential $\psi$, assuming the limit cycle occurs at $q=$ $\sqrt{q_{1}^{2}+q_{2}^{2}}=1$ :

$$
\begin{aligned}
S & =\frac{\left(q^{2}-1\right)^{2}}{\left(q^{2}-1\right)^{2}+1}\left(\begin{array}{ll}
1 & 0 \\
0 & 1
\end{array}\right) \\
T & =(q-1) \frac{q^{2}}{\left(q^{2}-1\right)^{2}+1}\left(\begin{array}{ll}
0 & -1 \\
1 & 0
\end{array}\right) \\
\psi & =\frac{1}{2}(q-1)^{2}
\end{aligned}
$$

The potential $\psi$ given in Eq.(7) is rotational symmetric in the state space. It has a local maximum $\psi=1 / 2$ at $q=0$, which is a fixed point, and the minimum $\psi=0$ at $q=1$, which is a cycle in the state space. Hence the potential takes the shape of Mexican hat type.
Evidently, if the friction matrix $S$ would be zero, the dynamical trajectory of the massless particle would move along the equal potential contour determined by the initial condition, which would be a cycle according to above chosen potential. In the presence of nonzero friction matrix, this is not true. What will be our concern is the behavior near the minimum of the potential function: When $q$ is sufficiently close to 1 , does the particle trajectory asymptotically approach the cycle of $q=1$ and eventually coincide with it? If the answer is positive, we have a limit cycle dynamics. We will demonstrate below that it is indeed possible.

For a deterministic dynamics, we can set $\epsilon=0$ in Eq.(1) and (2): setting the stochastic force to be zero. The dynamical equation can then be rewritten as

$$
\dot{\mathbf{q}}=-[S(\mathbf{q}, t)+T(\mathbf{q}, t)]^{-1} \nabla \psi(\mathbf{q}, t)
$$

With the choice of Eqs. (5-7), we have

$$
\begin{aligned}
{[S+T]^{-1}=} & \frac{1}{\operatorname{det}(S+T)}\left[\frac{\left(q^{2}-1\right)^{2}}{\left(q^{2}-1\right)^{2}+1}\left(\begin{array}{ll}
1 & 0 \\
0 & 1
\end{array}\right)\right. \\
& \left.\left.-(q-1) \frac{q^{2}}{\left(q^{2}-1\right)^{2}+1}\right)\left(\begin{array}{ll}
0 & -1 \\
1 & 0
\end{array}\right)\right]
\end{aligned}
$$

and $\operatorname{det}(S+T)=\left[\left(q^{2}-1\right)^{2} /\left(\left(q^{2}-1\right)^{2}+1\right)\right]^{2}+[(q-$ $1)^{2} q^{4} /\left[\left(q^{2}-1\right)^{2}+1\right]^{2}$. Near $q=1$, we have

$$
\begin{aligned}
{[S+T]^{-1}=} & \frac{1}{q-1}\left[-(1-2(q-1))\left(\begin{array}{ll}
0 & -1 \\
1 & 0
\end{array}\right)\right. \\
& \left.\left.+4(q-1)\left(\begin{array}{ll}
0 & -1 \\
1 & 0
\end{array}\right)+O\left((q-1)^{2}\right)\right] 10\right)
\end{aligned}
$$

In terms of radial coordinate $q$ and azimuth angle $\theta$ in the polar coordinate representation of the state space, using the small parameter expansion given in Eq.(10) following Eq.(8) we have, to the order of $q-1$,

$$
\begin{aligned}
\dot{q} & =-4(q-1) \\
\dot{\theta} & =1-2 \frac{q-1}{q}
\end{aligned}
$$

The solution is

$$
\begin{aligned}
q(t)= & 1+\delta q_{0} \exp \{-4 t\} \\
\theta(t)= & \theta_{0}+t+\frac{1}{4} \ln \frac{1+\delta q_{0} \exp \{-4 t\}}{1+\delta q_{0}} \\
& +\frac{1}{2} \ln \left(1+\delta q_{0} \exp \{-4 t\}\right)
\end{aligned}
$$

Here $\delta q_{0}\left(\left|\delta q_{0}\right|<<1\right)$ is the starting radial position of the particle measured from $q=1$ and $\theta_{0}$ its the starting azimuth angle. Indeed, the solution demonstrates the asymptotical approaching to the cycle $q=1$, and the motion never stops. Unstable and metable limit cycles may be constructed in the similar manner.

Though above construction does show that based on the knowledge of physical sciences one can construct limit 
cycle with a potential, the example of Eqs.(5-7) appears contrived. We should, however, point out that there are several generic features in our construction. First, to have an indefinitely motion on a closed trajectory, because of energy conservation, the friction, or better the friction matrix here, must be zero. Second, because of the asymptotic motion is on the equal potential contour, a Lorentz force type must exist to keep the motion on the contour. This means that the antisymmetric matrix should be finite along the equal motion contour when the potential gradient is finite. The speed of the massless particle moving along the contour will be determined by the ratio of the strength of the Lorentz like force to that of the gradient of the potential. Third, because the limit cycle should be robust: Small parameter changes should only have a small effect on the limit cycle, the (stable) limit cycle should be at the minimum of the potential. As a consequence, the potential gradient at the maximum is zero, which would imply that the friction matrix must go to zero faster than that of the potential gradient when approaching to the maximum to avoid the potential taking singular values. This means that at the limit cycle the dynamics is conserved. All those features are explicitly implemented in the choice, Eqs.(5-7).

Two additional remarks are in order. For simplicity of calculation we have chosen the friction matrix to be a constant matrix in Eq.(5). One can check that any positive definite symmetric matrix can lead to above same conclusion, as long as its strength goes to zero in a higher order comparing to that of the potential gradient. The second remark is that although $S$ is zero when approaching to the limit cycle, $[S+T]^{-1}+[S-T]^{-1}$ is not, on which we will come back later when discussing the diffusion matrix in section V.

\section{LIMIT CYCLE: SIMPLE CASE IN DYNAMICAL SYSTEMS}

The demonstration of the co-existence of limit cycle with potential in section II may appear special: The particle moves along the potential minimum with both zero friction and zero transverse matrices, a nice picture from physical sciences. A question naturally arises that whether or not in a typical limit cycle in dynamical systems a potential can be constructed. We demonstrate in this section that the answer is a straightforward yes.

It has been suggested that a typical and simple limit cycle in two dimensions would take following dynamical equation in a polar coordinate representation ${ }^{12}$ :

$$
\begin{aligned}
& \dot{q}=R(q) \\
& \dot{\theta}=\Phi(q)
\end{aligned}
$$

Here the smooth functions $R, \Phi$ have properties $R(q=$ $1)=0$ is a fixed point in the radial coordinate and $\Phi(q=$ $1)=$ constant . It is mathematically possible that any limit cycle in two dimension can be deformed into a cycle, and, at least near this limit cycle, the dynamical equation can be mapped into above form by a nonlinear coordinate transformation.

The comparison between Eqs. $(11,12)$ and Eqs. $(15,16)$ immediately suggests what considered in section II is just such a typical limit cycle. Hence, we can conclude that the potential and limit cycle coexist for such cases.

Nevertheless, we should point out an interesting paradoxical feature. On one side it is known from the theory of dynamical systems that a limit cycle is robust ${ }^{3}$. Any small parameter change in the equation would not lead to its disappearing. On other side, from the demonstration in section II which is based on the understanding from physical sciences, the existence of the limit cycle is a result of a very delicate balance between all dynamical elements: The friction matrix, the gradient of potential function, and the antisymmetric matrix are all zero at the limit cycle. How this feature would play a role in our better understanding limit cycle and its control will be an interesting problem for further exploration.

\section{GENERAL CONSTRUCTION OF EVOLUTION POTENTIAL}

A particular challenging and careful reader would observe that we have not demonstrated the potential for a general limit cycle in arbitrary dimensions. Hence there might exist a type of limit cycle, which will not be ones such as discussed above, such that a potential might not exist. We have been unable to give an explicit demonstration for the existence of potential for a most generic limit cycle (periodic attractors). However, we point out that such a discussion has been done for a generic fixed point (point attractors $)^{13}$. Furthermore, we do have a generic construction of potential for a general situation. This construction suggests it is possible to do so generally for arbitrary limit cycles. The general method has been presented elsewhere ${ }^{2}$. Here we outline its key ideas to make the present demonstration complete.

We restore the stochastic force back into the general equation in dynamical systems and consider an arbitrary dimension $n$ instead merely two dimensions:

$$
\dot{\mathbf{q}}=\mathbf{f}(\mathbf{q}, t)+\zeta(\mathbf{q}, t) .
$$

Here $\mathbf{f}(\mathbf{q}, t)$ is the deterministic nonlinear drive of the system and the stochastic drive is $\zeta(\mathbf{q}, t)$, which differs from that in Eq.(1) but comes from the same dynamical source. For simplicity we will assume that $\mathbf{f}$ is a smooth function whenever needed. To be consistent with Eq.(1) and (2), the stochastic drive in Eq.(17) is also assumed to be Gaussian and white with the variance,

$$
\left\langle\zeta(\mathbf{q}, t) \zeta^{\tau}\left(\mathbf{q}, t^{\prime}\right)\right\rangle=2 D(\mathbf{q}, t) \epsilon \delta\left(t-t^{\prime}\right),
$$

and zero mean, $\langle\zeta(\mathbf{q}, t)\rangle=0$. The matrix $D$ is the diffusion matrix.

Assuming that both Eq.(1) and (17) describe the same dynamics. It is rather easy to derive Eq.(17) from Eq.(1). 
The difficult part is to derive Eq.(1) from Eq.(17). Using Eq.(17) to eliminate $\dot{\mathbf{q}}$ in Eq.(1), and noticing that the dynamics of noise and the state variable behave independently, we have, the deterministic part,

$$
[S(\mathbf{q}, t)+T(\mathbf{q}, t)] \mathbf{f}(\mathbf{q}, t)=-\nabla \psi(\mathbf{q}, t),
$$

and the stochastic part,

$$
[S(\mathbf{q}, t)+T(\mathbf{q}, t)] \zeta(\mathbf{q}, t)=\xi(\mathbf{q}, t)
$$

Those two equations suggest a rotation in state space.

Multiplying Eq.(20) by its own transpose of each side and carrying out the average over stochastic drive, we have

$$
[S(\mathbf{q}, t)+T(\mathbf{q}, t)] D(\mathbf{q}, t)[S(\mathbf{q}, t)-T(\mathbf{q}, t)]=S(\mathbf{q}, t) .
$$

In obtaining Eq.(21) we have also used Eq.(2) and (18). Eq. (21) gives $n(n+1) / 2$ conditions because it is symmetric under the transpose operation.

Using the property of the potential $\psi: \nabla \times \nabla \psi=0$ $\left[(\nabla \times \nabla \psi)_{i j}=\left(\nabla_{i} \nabla_{j}-\nabla_{i} \nabla_{j}\right) \psi\right]$, Eq.(19) leads to

$$
\nabla \times[[S(\mathbf{q}, t)+T(\mathbf{q}, t)] \mathbf{f}(\mathbf{q})]=0
$$

which gives $n(n-1) / 2$ conditions because the antisymmetric condition implied.

Combining both Eq.(21) and (22), we have total $n^{2}$ conditions, which is exactly the number to determine the $n \times n$ matrix $[S(\mathbf{q}, t)+T(\mathbf{q}, t)]$, when supplemented by appropriate boundary condition needed by the partial differential equations of Eq.(22). This additional boundary condition should be the delicate balance condition discussed in section II and III: When approaching the limit cycle, the friction matrix must approach to zero faster than the potential gradient as well as the antisymmetric matrix. Once $[S+T]$ is known, it is straightforward to obtain the potential according to Eq.(19), hence construct Eq.(1) and (2) from Eq.(17) and (18).

The general existence of the potential implies that indeed the energy landscape concept in physical sciences should be one of the most important concepts in dynamics systems, too. Thus, not only the potential exist in periodic attractors, may also in the strange attractors. This would have implications in other fields where dynamical behaviors are the primary concerns. For example, we have shown elsewhere ${ }^{14}$ that the mathematical structure of population genetics can be formulated in such a way to incorporate the important concept of potential.

\section{DISCUSSIONS}

There are two mathematical subtleties. First, it is known that even with a limit cycle the dynamics is dissipative, reflecting by the fact that in general $\nabla \cdot \mathbf{f} \neq 0$, where $f$ is defined by Eq.(17). This is also expressed by the fact that the so-called diffusion matrix, $D$ defined in
Eq.(18), is finite even at the limit cycle. Because it is dissipative, it would be difficult to conceive a constant potential (or a Lyapunov function) along the limit cycle on which the dynamics repeats itself indefinitely. The delicate point is that, as shown in our above demonstration, that the friction matrix $S$ is zero along the limit cycle does not implies the diffusion matrix $D$ is zero. In fact, it is finite according to Eq.(21):

$$
D=\frac{1}{2}\left[(S+T)^{-1}+(S-T)^{-1}\right] .
$$

An interesting and direct implication is that the statement that one cannot construct Hopfield potential (or Lyapunov function) in limit cycle in computing by attractors in the neural networks ${ }^{4}$ is not true.

We come to the second point. For deterministic dynamics, if one finds one Lyaponov function, one finds many. This is illustrated by the present construction that additional information from the noise is needed: different diffusion matrix would lead to different potential function. Hence, this may provide a method to select best suitable Lyapunov function or potential function to ones own problem by choosing appropriate form the diffusion matrix.

It is perhaps worthwhile to point out that the typical gradient systems in dynamical systems theory corresponds to the zero transverse matrix, $T=0$, in the present construction. No limit cycle is possible in this case. In dynamics described by gradient systems the trajectory could follow the most rapid descendant path along the landscape defined by the potential. In this case it is easy to identify the potential as the landscape function. For a general dynamics where the transverse matrix is not zero, the trajectory would not follow the most rapid descendant route along the potential, as expressed by Eq.(1). Nevertheless, the meaning of the potential remains the same as that in gradient systems: determining the final distribution.

\section{CONCLUSIONS}

Based on the demonstrations in section II-IV, we conclude that a potential can coexists with limit cycle. Such a potential determines the final distribution according to a Boltzmann-Gibbs distribution. This may provide an alternative perspective to study the metastability of limit cycle from Kramers potential landscape point of view. In regarding to computing by attractors in neural networks, the present article shows that the Hopfield potential exists for periodic attractors.

Acknowledgement: We thank M. Dykman and $H$. Qian for critical discussions. This work was supported in part by a USA NIH grant under HG002894. 
1 Linder, B., Garcia-Ojalvo, J., Neiman, A., and Schimansky-Geier, L. (2004) Effects of noise in excitable systems. Phys. Rep. 382: 321-424.

2 Ao, P. (2004) Potential in stochastic differential equations: novel construction. J. Phys. A 37: L25-L30.

${ }^{3}$ Guckenheimer, J. and Holmes, P. (1997) Nonlinear Oscillations, Dynamical Systems, and Bifurcations of Vector Fields. Springer-Verlag, Berlin.

4 Hertz, J. (2003) Computing with attractors. In The Handbook of Brain Theory and Neural Networks. 2nd edition. Edited by M.A. Arbib. MIT Press, Cambridge. pp248-252.

${ }^{5}$ Berglund, N., and B. Gentz (2004) On the noise-induced passage through an unstable periodic orbit I: two level model. J. Stat. Phys. 114: 1577-1618.

6 Smelyanskiy, V.N., Dykman, M.I., and Maier, R.S. (1997) Topological features of large fluctuations to the interior of a limit cycle. Phys. Rev. E 55: 2369-2391; correction ibid 56: 2332-2332.

7 Bandrivskyy, A., Beri, S., Luchinsky, D.G., Mannella, R., and McClintock P.V.E. (2003) Fast Monte Carlo simulations and singularities in the probability distributions of nonequilibrium systems. Phys. Rev. Lett. 90: 210201-1-4.

8 Jaffe, C., Ross, S.D., Lo, M.W., Marsden, J., Farrelly, D., and Uzer, T. (2002) Statistical theory of asteroid escape rates. Phys. Rev. Lett. 89: 011101-1-4.

${ }^{9}$ H.A. Kramers (1940) Brownian motion in a field of force and the diffusion model of chemical reactions. Physica 7: 284-304.

10 P. Hanggi, P. Talkner, and M. Borkevic (1990) Reactionrate theory: fifty years after Kramers. Rev. Mod. Phys. 62: 251-341.

11 Goldstein, H. (1980) Classical Mechanics. 2nd edition. Addison-Wesley, Reading.

12 Murray, J.D. (2002) Mathematical Biology I: an introduction. 3rd edition. Springer, New York. pp280.

13 Kwon, C., Ao, P. and Thouless, D. J. (2003). Structure of stochastic dynamics near fixed points (submitted. Manuscript available upon request).

14 Ao, P. (2004) Mathematical structure of evolutionary theory. (submitted. Available at the e-print archive: q-bio/0403020) 